\title{
Internet of Things in Psoriasis Assessment and Treatment
}

\author{
Simona Maria Banu, Laura Mădălina Dascălu, and Gheorghe Toacșe \\ Transilvania University of Brașov, România, \\ Department of Electronics and Computers, \\ Faculty of Electrical Engineering and Computer Science \\ \{simonam.banu\}@gmail.com, \{madalina.dascalu, toacseg\}@unitbv.ro
}

\begin{abstract}
Within the Internet of Things (IoT) paradigm, an everyday object can be transformed into a smart object, able to sense, interpret and react to the environment. IoT is bringing new ways of communicating between people and things (objects) to reach common goals, bringing a high impact on everydaylife. The aim of this paper is to present how people with psoriasis and their doctors can benefit from the IoT advantages. There is presented a proposed system for surveillance and treatment plan for patients suffering from psoriasis using assisted IoT and Computer Vision technologies.
\end{abstract}

Keywords: Internet of Things, psoriasis severity, Expert System.

\section{$1 \quad$ Introduction}

The Internet of Things (IoT) is meant to refer to a vision of an Internet where there is a strong connectivity between anyone and anything, and takes place anywhere and anytime. It envisions a future in which physical and digital things can be linked to enable and support new classes of applications and services. There are many fields in which IoT has started to bring important contribution: health and e-health, domotics, monitoring systems, logistics [1].

The use of cross-layer communication schemes to offer adaptive solutions for the IoT is motivated by the high heterogeneity in the hardware capabilities and the communication requirements among things. IoT permits a global communication on existing layered solutions. It also provides efficient and reliable end-to-end communication. Among the possible IoT domains and corresponding applications, we can consider the following: healthcare (tracking, identification, authentication, data collection), smart environments (smart homes, industrial plants), transportation and logistics (assisted driving, augmented maps), personal and social (social networking, historical queries) [2].

There is a growing demand for more advanced and better healthcare solutions with the focus on: a) decrease of healthcare costs; b) a need for better and more efficient outcomes. Our objective is to bring some preliminary ideas/evidence of improving the quality of healthcare and/or reduce the cost of it. 
Psoriasis is a skin condition that develops when the immune system malfunctions. It is estimated that about $5 \%$ of the global population have this genetically-determined chronic skin disease [3]. People with psoriasis may have discomfort in the form of pain and itching, and emotional distress (see Fig. 1).
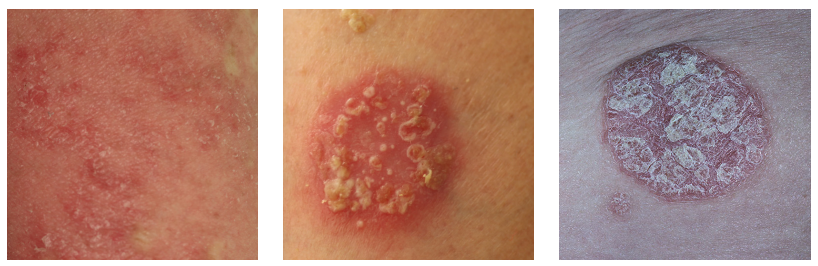

Fig. 1. Different psoriasis lesions photographed under different illumination conditions

In the next sections we will cover the following aspects: (i) technical contribution to the Internet of Things; (ii) related work in both Internet of Things technology and also in Expert Systems for dermatology (psoriasis in particular); (iii) detail presentation of the proposed system; (iv) discussion of preliminary results; and (v) final conclusions and future work.

\section{Relationship to Internet of Things}

In this paper, we propose a prototype that aims to integrate physical and digital things (IoT technology) into the everyday life of patients and their medical doctors. We try to respond to the needs of patients and healthcare professionals by proposing a new expert system that envisions the IoT paradigm. This expert system will objectively assess the erythema (degree of redness) for psoriasis severity evaluation. The IoT solution is meant to give guidance to medical doctors in prescribing the best treatment for patients suffering from psoriasis disease.

The system's architecture is based on smart objects (things) and is designed to meet primarily the expectations of patients and doctors. The patients would benefit from a high quality personalized healthcare on an equal access basis, regardless of their social status and location, with full respect of their privacy. Also they can stay at home as much as possible due to the ability of the system to collect data provided by the patients themselves (this data being further evaluated by the doctor who will have access to it through an intelligent web application). On the other hand, the healthcare professional would be able to follow up any development in the patient's health condition at home and also, would have access to the health information of other patients through this healthcare system of sharing information between medical facilities (by having an RFID tag reader present which would be able to read the tag inserted in the patient's smart phone). 


\section{Related Work}

Psoriasis is a long lasting skin disease depicted when patches of skin become inflamed and scales develop. Psoriasis Area and Severity Index (PASI) [4] is an evaluation technique that measures the overall psoriasis severity and coverage (see Fig. 2). PASI is the most used measure in clinical trials when assessing the psoriasis severity and therefore, giving the proper treatment.

The PASI score computation is done in a subjectively manner by evaluating three characteristics (erythema, induration and scaliness) of psoriatic lesions (see Fig. 2). The Abbott PASI meter tries to standardize these psoriasis characteristics into four types (Mild, Moderate, Severe and Very severe).

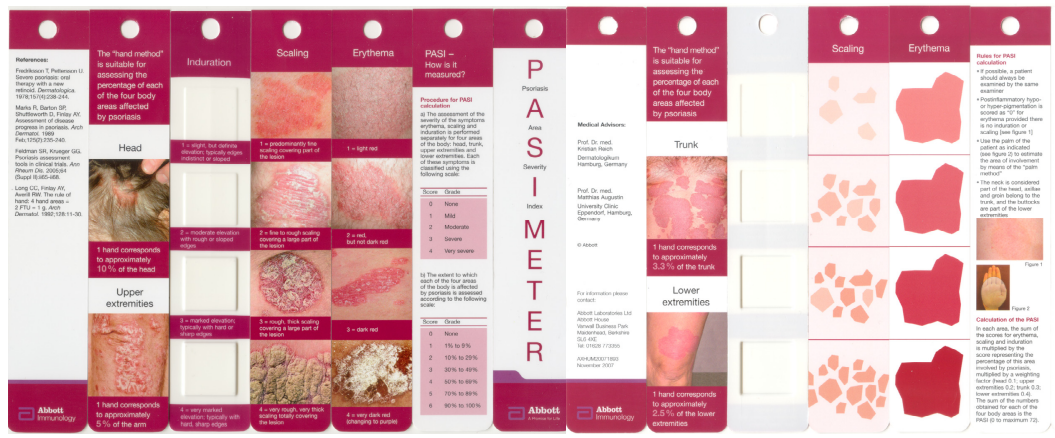

Fig. 2. Abbott PASI meter

There are several papers that give a solution to this subjective evaluation by objectively assess the lesion thickness and erythema for psoriasis severity evaluation [5], [6].

Expert Systems are a sub-type of Artificial Intelligence systems used in clinical diagnosis. They contain medical knowledge and are able to output logical conclusions given as entry point specific data from individual patients. Although there are many variations, the knowledge within an expert system is typically represented by a set of rules or criteria.

Recent research presents different expert systems for differential diagnosis of erythemato-squamous diseases. For example, the paper presented in [7] aimed the implementation of a visual tool for differential diagnosis of skin diseases, which is a difficult problem in dermatology, because several diseases share almost the same clinical features of erythema and scaling.

The main objective of our expert system is not to make a differential diagnosis, but to classify the already known psoriasis disease in order to further help the dermatologist in giving the correct treatment.

The RFID technology (Radio Frequency IDentification) can be used to put identification labels (e,g. tags) into objects (tablets, mobile phones). If the consecrated reader of these tags is in next proximity of the tagged object, the information related to it can be accessed without any physical contact. In this way, the whole process of 
patient's identification and database access is simplified and the timings are appreciable reduced [8].

There are several medical applications that make use of RFID technology [9], [10], [11].

In the following section we will present our proposed system used for surveillance and treatment plan for patients suffering from psoriasis disease.

\section{The Proposed System}

In the Romanian healthcare sector, the information related to each individual patient is scattered among several hospitals and medical clinics, with no connection among them. There is a major lack of interoperability due to no standardized content related to patients' medical records. The data provided in each database is sometimes incomplete and there is no way to update it by synchronizing it with different other databases from other cities (or even other medical clinics from within the same city).

Considering the above-mentioned disadvantages we propose in this paper an expert system that can be used for the objective assessment of psoriasis. This system is integrated in a bigger IoT architecture which includes three main layers: a perception (or sensor) layer, a network layer and an application layer. The perception layer gathers information from objects which are transmitted further to the application layer through the network layer.

The basic idea behind IoT is the fact that we are surrounded by many things or objects which interact with each other to reach common goals [12]. Objects or things can be considered the following: RFID tags, sensors, actuators, mobile phones. A series of objects that appear in the proposed system are presented in Fig. 3.

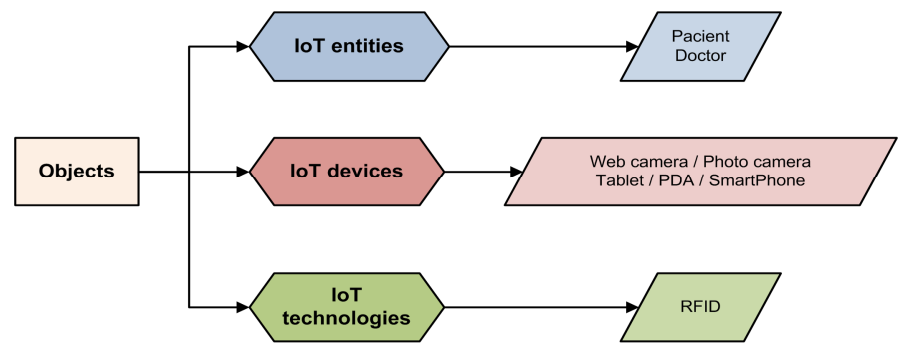

Fig. 3. Objects (things) present in the proposed system

The proposed IoT architecture is presented in Fig. 4. The system is based on the RFID technology which includes the following main components:

- RFID readers placed in hospitals, medical clinics etc.

- Smart phones equipped with RFID tags;

- An EMR (Electronic Medical Record) server for storing the medical records of individual patients regardless their location;

- A HL7 server for interoperability with other clinical software. 


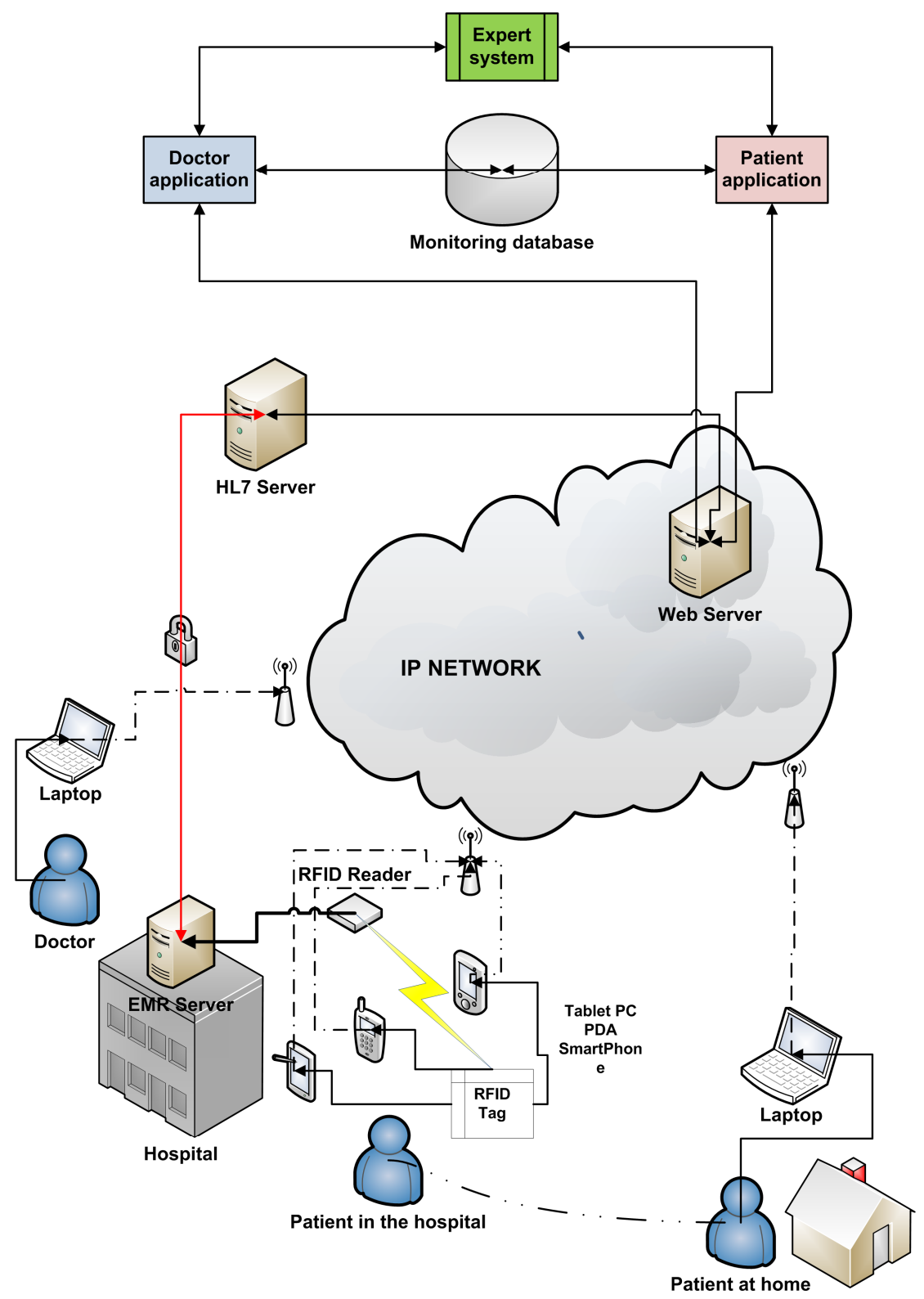

Fig. 4. General view of the IoT architecture for the proposed system

Even though the proposed RFID-based system is similar to the SIMOPAC system described in [13] the application types and the main idea are different. Therefore, our proposed expert system has a more specific task, by objectively evaluating the erythema severity of psoriasis lesions regardless the location of the patient (at a 
clinical facility or at home) and also by updating the medical records through the intelligent applications which can be accessed by doctors and also by patients.

The ability to integrate and exchange information among similar systems will be achieved through HL7 (Health Level Seven) standards. This type of standard is promoted in the healthcare industry due to its quality, accuracy, and efficiency characteristics [14].

In the application layer the two types of applications (doctor's and patient's application) are present. They are connected to the expert system (see Fig. 5) which returns the degree of erythema for the psoriasis lesions. The psoriasis lesions are first photographed by the patient at home or by the doctor in the hospital. The images are then uploaded through the specific applications onto the system and the expert system is processing this input. From the answer given by the expert system the doctor can give the proper treatment and also, can update the medical data related to each patient.

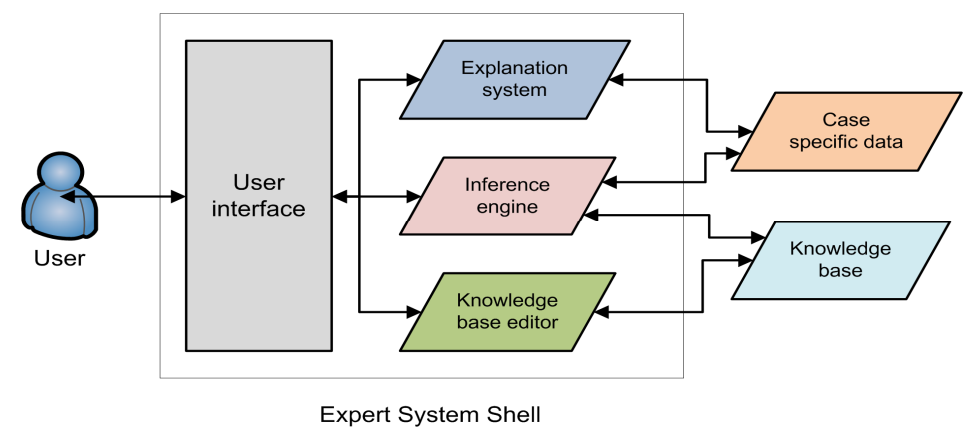

Fig. 5. Expert System architecture

The presented IoT solution enables the system management, respectively the disease pathway. It brings transparency in the responsibility of impact, it reduces the subjective visual analyze of psoriasis by doctors and also gives guidance during treatment plan.

The main benefits of the proposed system consist in: immediate access to data, the possibility of transmitting data when it is available (patients are uploading the pictures whenever they want and doctors can access uploads whenever they want), direct feedback on the treatment efficiency, the time of every day visual diagnosis during the treatment is reduced, all the process of clinical pathway is more efficient and helps the patient to comply with his/her treatment routine.

The main risks that might appear are the information accuracy, which depends mostly on the quality of the image uploaded by the patients and the need of more evidence (the induration or thickness measure for the psoriasis lesion which can be evaluated only by the medical doctor) for treating the disease.

\section{Discussions of Results}

In order to develop an expert system that can be used in the dermatology field we have to first classify the psoriasis severity for different lesions. The input to our expert 
system is composed of digital images depicting psoriatic lesions. Due to the different illumination conditions a color correction step is needed.

In the research done in [6] the authors propose an approach for objective assessment of the erythema degree. Their approach comprises of three steps: (i) reference-based color correction, (ii) lesion segmentation and (iii) erythema classification and ranking. Due to the correction step, the color segmentation and classification could be correctly performed, and also, the specialist could assess the erythema severity with less ambiguity when being presented with images of lesion images previously color corrected.

We intend to use a similar approach when developing our psoriasis evaluation expert system. Having a limited set of images containing lesions of psoriasis we are in the process of enlarging our database with different other images (test cases) to get a higher accuracy when objectively assess the erythema severity.

\section{Conclusions and Future Work}

We strongly believe that the IoT can offer assistance, support and follow-up of patients which are suffering from psoriasis symptoms, both in hospitals/treatment clinics and at distance (home).

We have presented in this paper a solution for using IoT for improving the quality of healthcare in psoriasis diagnosis and treatment. There were presented two possible applications that can be used both by patients and doctors. The applications show how patients can achieve a better quality of life while treating the disease, during the prescript treatment.

Using the proposed Internet platform the costs for transferring patients from one hospital to another, for diagnosis and/or treatment, is reduced. Also, the system can guarantee the privacy and security of patient's records.

In the future, we intend to implement all the necessary things for bringing to life the system for surveillance and treatment plan for patients suffering from psoriasis disease.

Acknowledgments. This paper is partially supported by the Sectoral Operational Programme Human Resources Development (SOP HRD), ID76945 financed from the European Social Fund and by the Romanian Government.

\section{References}

1. Gusmeroli, S., Haller, S., Harrison, M., Kalaboukas, K., Tomasella, M., Vermesan, O., Vogt, H., Wouters, K.: Vision and challenges for realizing the internet of things. In: Friess, P., Guillemin, P., Sundmaeker, H., Woelfflé, S. (eds.) European Commission (2010)

2. Atzori, L., Iera, A., Morabito, G.: The internet of things: a survey. Computer Networks (Elsevier) Journal 54, 2787-2805 (2010)

3. Raychaudhuri, S., Farber, E.: The prevalence of psoriasis in the world. Journal of European Academy of Dermatology and Venerology 15(1) (January 2001) 
4. Langley, R.G., Ellis, C.N.: Evaluating psoriasis with psoriasis area and severity index, psoriasis global assessment, and lattice system physician's global assessment. Journal of the American Academy of Dermatology 51(4), 563-569 (2004)

5. Ahmad Fadzil, M.H., Fitriyah, H., Prakasa, E., Nugroho, H., Hussein, S.H., Affandi, A.M.: Objective assessment of psoriasis lesion thickness for PASI scoring using $3 \mathrm{~d}$ digital imaging. Proceedings of World Academy of Science, Engineering and Technology 63, 109-115 (2010)

6. Ivanovici, M., Banu, S.M., Irimie, M., Căliman, A., Richard, N.: Objective assessment of erythema for psoriasis severity evaluation. Submitted to International Symposium on Biomedical Imaging: From Nano to Macro (2013)

7. Güvenir, H.A., Emeksiz, N.: An expert system for the differential diagnosis of erythematosquamous diseases. Expert Systems with Applications 18, 43-49 (2000)

8. Laranjo, I., Macedo, J., Santos, A.: Internet of Things for Medication Control: Service Implementation and Testing. Procedia Technology 5, 777-786 (2012)

9. McCall, C., Maynes, B., Zou, C.C., Zhang, N.J.: RMAIS: RFID-based medication Adherence Intelligence System. In: 2010 Annual International Conference of the IEEE Engineering in Medicine and Biology Society (EMBC), pp. 3768-3771 (2010)

10. Yeung, C.L., Kwok, S.K., Mui, H.C.: An Investigation of an RFID-based Patient-tracking and Mobile Alert System. International Journal of Engineering Business Management (2011)

11. Vilamovska, A.M., Hattziandreu, E., Schindler, R., Van Oranje, C., DeVries, H., Krapelse, J.: RFID Application in Healthcare - Scoping and Identifying Areas for RFID Deployment in Healthcare Delivery. RAND Europe (February 2009)

12. Giusto, D., Iera, A., Morabito, G., Atzori, L.: The Internet of Things. Springer (2010)

13. Turcu, C., Cerlinca, T., Turcu, C., Cerlinca, M., Prodan, R.: An RFID and multi-agent based system for improving efficiency in patient identification and monitoring. WSEAS Transactions on Information Science and Applications 6(11), 1792-1801 (2009)

14. Shaver, D.: HL7 101: A Beginner's Guide. For the Record 19(1), 22 (2007), http://www. fortherecordmag.com/archives/ftr_01082007p22.shtm1 